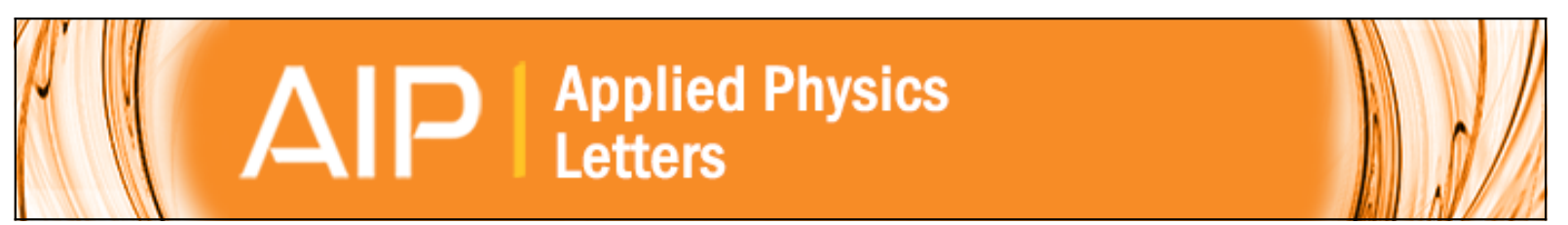

Simultaneous electrical and optical readout of graphene-coated high $\mathbf{Q}$ silicon nitride resonators

V. P. Adiga, R. De Alba, I. R. Storch, P. A. Yu, B. llic, R. A. Barton, S. Lee, J. Hone, P. L. McEuen, J. M. Parpia, and H. G. Craighead

Citation: Applied Physics Letters 103, 143103 (2013); doi: 10.1063/1.4823457

View online: http://dx.doi.org/10.1063/1.4823457

View Table of Contents: http://scitation.aip.org/content/aip/journal/apl/103/14?ver=pdfcov

Published by the AIP Publishing

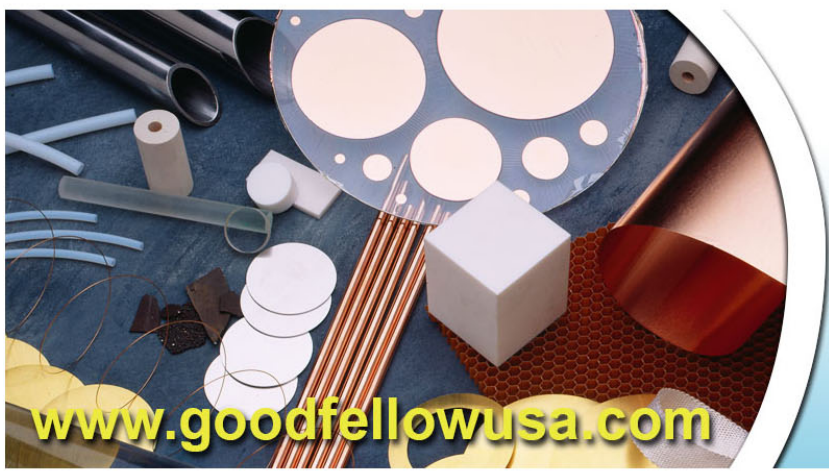

BODIFEl|OII

metals • ceramics • polymers composites • compounds • glasses

Save $5 \% \cdot$ Buy online 70,000 products $\cdot$ Fast shipping 


\title{
Simultaneous electrical and optical readout of graphene-coated high $Q$ silicon nitride resonators
}

\author{
V. P. Adiga, ${ }^{1, a)}$ R. De Alba, ${ }^{2}$ I. R. Storch, ${ }^{2}$ P. A. Yu, ${ }^{3}$ B. Ilic, ${ }^{4}$ R. A. Barton, ${ }^{1}$ S. Lee, ${ }^{5}$ J. Hone,${ }^{5}$ \\ P. L. McEuen, ${ }^{6}$ J. M. Parpia, ${ }^{2}$ and H. G. Craighead ${ }^{1, a)}$ \\ ${ }^{1}$ School of Applied and Engineering Physics, Cornell University, Ithaca, New York 14853, USA \\ ${ }^{2}$ Department of Physics, Cornell University, Ithaca, New York 14853, USA \\ ${ }^{3}$ Department of Chemical Engineering, California Institute of Technology, Pasadena, California 91125, USA \\ ${ }^{4}$ Cornell NanoScale Science \& Technology Facility, Ithaca, New York 14853, USA \\ ${ }^{5}$ Columbia University, New York, New York 14853, USA \\ ${ }^{6}$ Laboratory of Atomic and Solid State Physics and Kavli Institute at Cornell for Nanoscale Science, Ithaca, \\ New York 14853, USA
}

(Received 2 July 2013; accepted 10 September 2013; published online 30 September 2013)

\begin{abstract}
Resonant mechanics of high quality factor (Q) graphene coated silicon nitride devices have been explored using optical and electrical transduction schemes. With the addition of the graphene layer, we retain the desirable mechanical properties of silicon nitride but utilize the electrical and optical properties of graphene to transduce and tune the resonant motion by both optical and electrical means. By positioning the graphene-on-silicon-nitride drums in a tunable optical cavity, we observe position dependent damping and resonant frequency control of the devices due to optical absorption by graphene. (C) 2013 AIP Publishing LLC. [http://dx.doi.org/10.1063/1.4823457]
\end{abstract}

Resonant electromechanical systems ${ }^{1,2}$ and optomechanical systems ${ }^{3}$ with high quality factors have been studied for applications such as ultrasensitive force measurements and displacement sensing at the quantum limit. ${ }^{3}$ They have also found use in accelerometers and gyroscopes ${ }^{4}$ and show promise for resonant sensing applications. ${ }^{5,6}$ Silicon nitride has desirable mechanical properties for microelectromechanical devices (MEMS) and is relatively simple to fabricate. Ultrathin mechanical resonators made from silicon nitride have been explored for optomechanics, ${ }^{3}$ mass sensing, ${ }^{7}$ and force sensing ${ }^{8}$ because of their high mechanical quality factors ${ }^{9-11}$ $\left(\mathrm{Q}>10^{6}\right)$, low masses, and low spring constants. ${ }^{3,11}$ Recently, it has been shown that membrane Q can be enhanced by the right choice of tensile stress, resonator size, mode shape, and optimized fabrication techniques; ${ }^{9,10,12}$ quality factors of up to $4.4 \times 10^{6}$ can thus be achieved for a $15 \mathrm{~nm}$ thick silicon nitride membrane. ${ }^{9}$ Such large area, ultra-thin tensioned membranes are useful as optomechanical elements ${ }^{3,13}$ whose mechanical degrees of freedom can be easily controlled using light. ${ }^{3,11,13}$ However, because of the insulating nature of silicon nitride, some of the most desirable characteristics of these high-Q resonators can only be transduced optically. Electrical integration of these devices can be achieved through deposition of a thin conducting layer on the resonator surface. For metals, however, the thickness required to form a continuous layer results in significantly degraded Q and increased mass. ${ }^{14-16}$ Metallization also adds complexity in terms of stresses associated with thermal expansion mismatch, causing the freestanding structures to bend or buckle.

Graphene has been widely studied because of its unique electronic, ${ }^{17}$ optical, ${ }^{18}$ and mechanical properties. ${ }^{19}$ Its light mass and strong optical absorption make it an ideal candidate

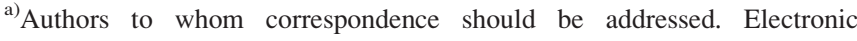
addresses: vpa8@cornell.edu and hgc1@cornell.edu
}

for achieving optomechanical coupling. ${ }^{13}$ Mechanical resonant devices have been constructed of monolayer graphene, ${ }^{20-24}$ but the mechanical quality factor, fabrication yield, and durability of these structures are limited. Hybrid silicon nitride-graphene ( $\mathrm{SiNG})$ devices that combine the properties of both materials would greatly expand the range of possible device applications, combining the desirable mechanical properties of silicon nitride with the electrical and optical properties of graphene. In this article, we demonstrate the electrical actuation of high stress silicon nitride membranes using monolayer graphene in a tunable Fabry-Perot cavity. We also present simultaneous detection of its resonant motion using both optical and electrical means enabling the comparison of the two detection schemes. Strong optical absorption in the atomic monolayer graphene ${ }^{18}$ enables photothermal interaction with the high-Q silicon nitride membrane, with associated frequency and damping tunability due to tension modulation in the nitride. The electrical detection of this optical interaction over the entire cavity detuning $(\mathrm{z} / \lambda)$ range is useful to understand the photothermal processes ${ }^{13}$ in these heterostructures; it enables us to decouple the resonant motion modulation due to optical absorption from the position-sensitive optical detection scheme. These frequencytunable optically and electrically coupled systems have applications including oscillators, filters, and sensors. ${ }^{25-27}$ Electrical integration of these high Q devices also enables us to understand mechanical nonlinearities ${ }^{28-30}$ and provide greater scope for quantum control and cooling. ${ }^{31}$

Silicon nitride-graphene square drums of side length $100 \mu \mathrm{m}-400 \mu \mathrm{m}$ were fabricated using potassium hydroxide $(\mathrm{KOH})$ etching of the backside of a silicon wafer. Chemical vapor deposition (CVD) grown graphene was transferred on top of a wafer containing suspended drums and patterned using optical lithography. Electrical contacts to these resonators were defined by patterning metal leads. ${ }^{32}$ These graphene on silicon nitride devices are placed in close 


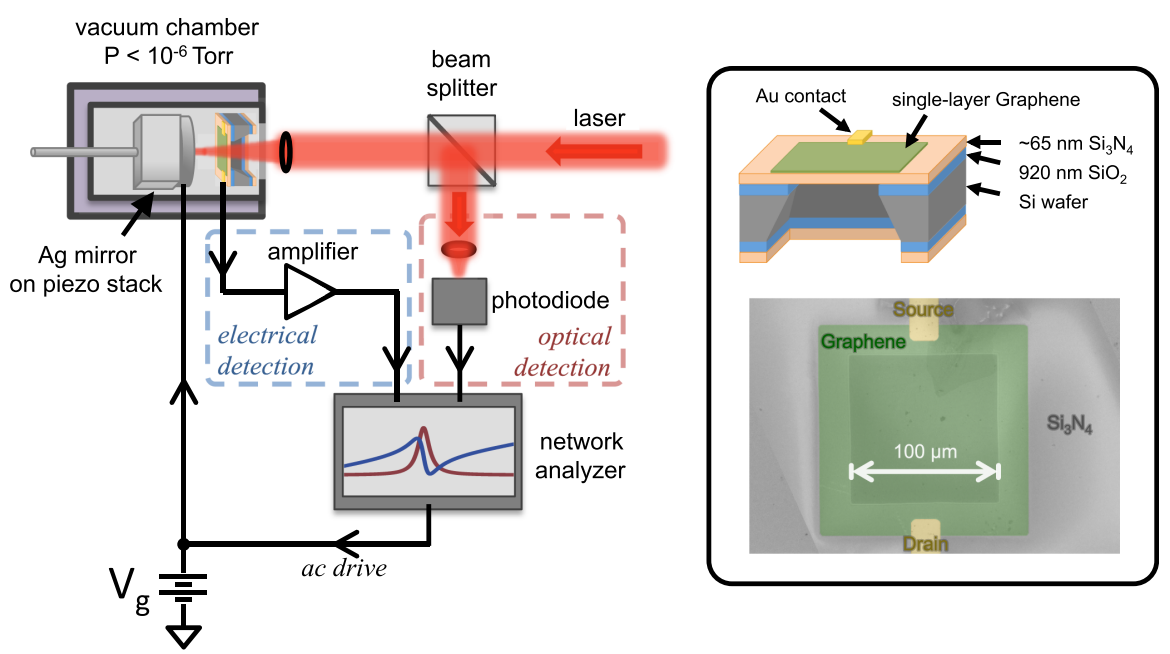

FIG. 1. Schematic of the experimental setup. Variation in the reflected light from a Fabry-Perot cavity formed between a graphene on silicon nitride membrane and a piezo-controlled metallic mirror is monitored by a fast photo diode. A gate voltage, $\mathrm{V}_{\mathrm{g}}$, is applied between the graphene and the metallic mirror to actuate the resonator; this voltage has a DC component for tuning and an AC component at the drive frequency. Measuring the capacitively coupled current provides a second means to readout mechanical motion. Inset: Combined scanning electron microscope (SEM) and optical micrograph of a typical SiNG membrane resonator showing the device layout. Scale bar indicates the suspended region.

proximity to a piezo-controlled metallic mirror that forms a tunable optical cavity. ${ }^{32}$ Optical detection involves detecting the change in the reflected laser light as the membrane moves in the low finesse $(F \approx 1.2)$ optical cavity formed by the membrane and mirror as shown in Figure 1. The metallic mirror used in this cavity also acts as a conductive electrode which is placed in close proximity to the resonator $(<60 \mu \mathrm{m})$, where we apply a bias voltage to actuate and tune the resonance of the SiNG membranes electrostatically under high vacuum conditions $\left(<2 \times 10^{-6}\right.$ Torr $)$. A fast photodiode and a network analyzer are used to measure the time-varying component of our reflected optical signal. This detected signal is proportional to both the amplitude of the membrane's motion $(\tilde{z}(\omega))$ and the change in cavity reflectance $(R(z))$ with respect to membrane position $(d R / d z) .{ }^{33}$ The amplitude of the capacitively driven membrane motion is given by

$$
\tilde{z}(\omega)=-\frac{1}{m_{e f f}} \frac{C_{g}}{d} V_{g} \tilde{V}_{g} \frac{1}{\omega_{0}^{2}-\omega^{2}+i \omega_{0} \omega / Q},
$$

where $C_{g}=\varepsilon_{0} A / d$ is the membrane-mirror capacitance, $m_{e f f}$ is the membrane's effective mass, and $A$ is the membrane area. $V_{g}, \tilde{V}_{g}$ are the DC gate voltage and AC drive voltage, respectively. $\omega_{0}=2 \pi f_{0}$ and $\omega=2 \pi f$ are the membrane resonant frequency and the drive frequency, respectively. Our electrical detection scheme involves the capacitive detection of membrane motion, ${ }^{32}$ and the observed signal $(\tilde{I})$ is given by $\tilde{I}=i \omega C_{t o t} \tilde{V}_{g}-i \omega \frac{\tilde{z}(\omega)}{d} C_{g} V_{g}$. The first term here corresponds to the total capacitive background $\left(C_{t o t}\right)$, due to the device $(\sim 1.5 \mathrm{pf})$ and all parasitic capacitance $(\sim 5 \mathrm{pf})$. The second term is sensitive to membrane motion.

Figure 2 shows the typical gate tuning of the resonant frequency, where the composite membrane only shows capacitive softening ${ }^{34}$ in the applied DC gate voltage range. At a given gate voltage, both optical and electrical resonant responses show a Lorentzian behavior (Figures 2(d) and 2(e)), allowing us to extract the fundamental frequency $\left(f_{0}=2.8 \mathrm{MHz}\right)$, the full width at half maximum $\Gamma$, and the quality factor $\left(Q=f_{0} / \Gamma\right)$ of the device. Electrically and optically detected signals give identical $Q$ and resonant frequency measurements (within fitting errors). For the fundamental mode of a tensioned square drum, the resonant frequency is given by $f_{0}=\frac{1}{L} \sqrt{\frac{\sigma}{2 \rho}}$, where $\sigma, \rho, L$ are stress, density, and side length of the resonator, respectively. This yields a tensile stress of $475 \mathrm{MPa}$ in our $100 \mu \mathrm{m}$ membrane. As such, quality factors of thin tensioned membranes scale approximately linearly with the aspect ratio (side length/thickness). ${ }^{9}$ We have measured quality factors of up to $\sim 70000$ for a $100 \mu \mathrm{m}^{2}$ graphene-on-silicon-nitride drum for the fundamental mode. A similar $300 \mu \mathrm{m}$ membrane yields $\mathrm{Q} \sim 250000$. Graphene
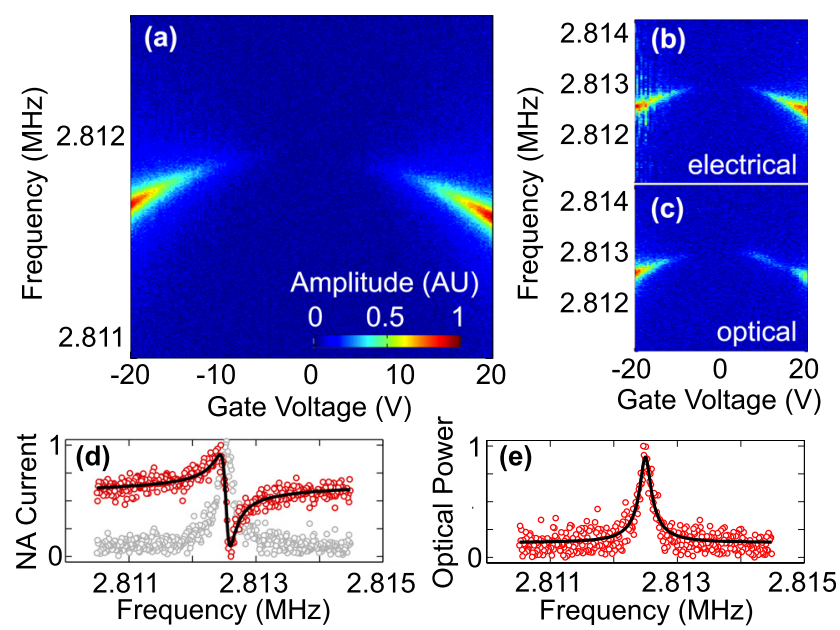

FIG. 2. (a) Gate tuning resonant frequency of the $100 \mu \mathrm{m}$ membrane, detected using electrical means with zero laser power. Amplitude is in color scale. The resonator shows capacitive softening in the measured voltage range. Gate tuning detected using both electrical (b) and optical (c) means at $100 \mu \mathrm{W}$ laser power. (d) Sample of raw electrical readout data with fit. Gray data points show the same data with the parasitic capacitance contribution subtracted, illustrating the Lorentzian signal as it appears in the color of (a) and (b). (e) Sample of optical readout data with fit. Fits give $f_{0}=2.8 \mathrm{MHz}$ and $\mathrm{Q}=17000$. 

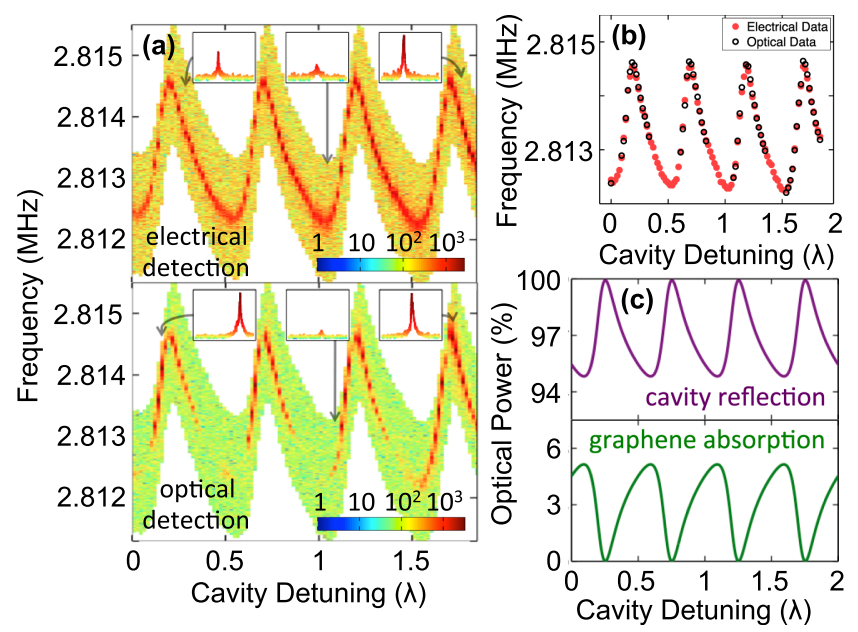

FIG. 3. (a) Electrical and optical detection of resonant frequency as a function of detuning of the cavity. Color scale indicates the amplitude of motion in log scale. While the electrical signal is continuous (capacitive background is subtracted from the data), there are positions in the cavity where optical signal disappears ( $d R / d z$ vanishes). (b) Overlaid resonance frequencies from fits of the electrical and optical readout data shown in (a). (c) Calculated reflectance of the optical cavity (purple) and absorption (green) by graphene as a function of detuning of the cavity.

contributes marginally to the observed mechanical damping of these structures. ${ }^{35}$

Figure 3 shows the optically and electrically detected resonant frequency response as the optical cavity is detuned by stepping the piezo controlled mirror toward the membrane at a fixed incident laser power $(0.2 \mathrm{~mW})$. The disappearance of the optical readout signal corresponds to the positions in the cavity where $d R / d z$ vanishes. We see a corresponding phase change in the optically detected signal. ${ }^{32}$ The electrically detected signal is continuous and shows an overall increase in signal as the mirror approaches the membrane. ${ }^{32}$ The optical detection scheme results in a better signal to noise ratio except near the points where the cavity reflectivity is close to its turning point. However, optical detection also is responsible for the associated photothermal interaction resulting in periodic variations in the resonant frequency. We model this interaction by calculating the cavity reflectance $R(z)$ and graphene absorption $A(z)$ (Figure 3(c)) in the cavity using a standard transfer matrix approach. ${ }^{32} A(z)$ in these calculations exceeds the well known value of $\pi \alpha \approx 2.3 \%$ due to the cavity effect, and the asymmetric cavity response is caused by reflections within the nitride layer. The slight offset of the nodes (corresponding to $d R / d z=0$ ) in the optically detected signal relative to the frequency extremes is indicative of additional losses in the cavity - attributed here to absorption by the Ag mirror. Figure 4(a) shows the electrically obtained resonant frequency as a function of mirror position for several values of the incident laser power, with corresponding fits based on the calculated optical absorption of graphene in our system (Figure $3(\mathrm{c})$ ). Nodal positions in the optical data were used to determine several cavity parameters in these fits based on $d R / d z{ }^{32}$ We observe that the magnitude of the frequency variation (defined as the peak-to-peak frequency shift) scales linearly with incident laser power (shown in Figure 4(b)).

Both optically and electrically obtained data suggest that the mechanism responsible for resonant frequency shifts
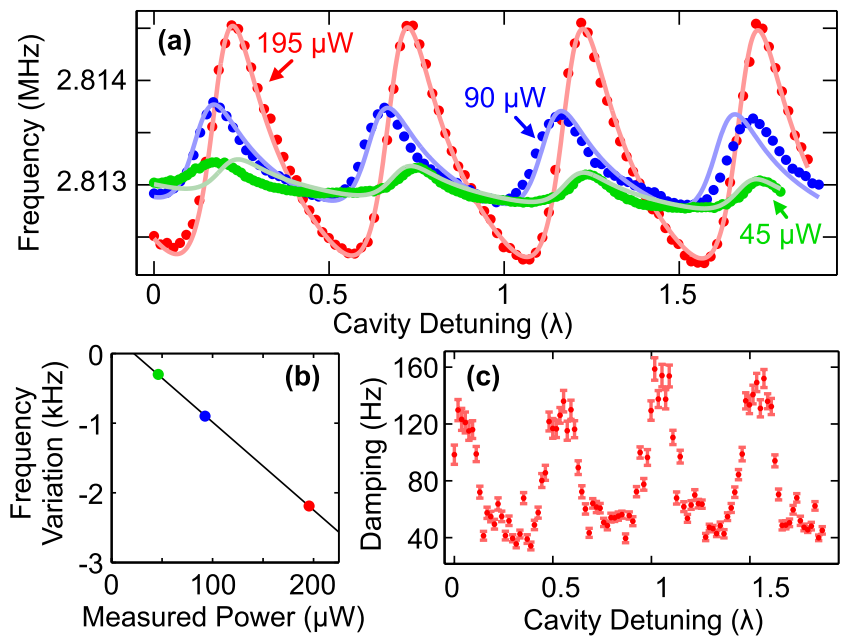

FIG. 4. (a) Resonant frequency of the membrane as a function of incident optical power and cavity detuning measured using electrical detection. Oscillations in the frequency are associated with optical absorption by the graphene and its effect on the tensile stress of the bilayer membrane. (b) Maximum frequency variation of the device as a function of incident laser power, with a linear fit. (c) Measured damping shifts at $195 \mu \mathrm{W}$ laser power, in part due to the photothermal feedback on the membrane.

in our devices is local heating in the membrane resulting from optical absorption by the graphene. Such heating leads to a lowering of the membrane's tensile stress through thermal expansion of the silicon nitride. In the low optical power limit, this results in a frequency shift that varies with temperature as $\frac{\Delta f}{f_{0}}=-\frac{E_{S i N} \alpha_{S i N} \Delta T}{2 \sigma}$, where $E_{S i N}$ and $\alpha_{S i N}$ are the Young's modulus and thermal expansion coefficient of nitride, and $\Delta T$ is the temperature shift due to optical heating. The numerator in this expression is the change in tension caused by expansion of the nitride and ignores contributions from graphene contraction since the graphene thickness is small compared to silicon nitride and has minimal affect on the overall mechanics. To lowest order, the temperature rise can be approximated by assuming a circular membrane and solving for the equilibrium heat flow radially outward from the laser spot. Including heat dissipation through both the graphene and the nitride, the steady state temperature difference between the membrane edge and the laser spot is $\Delta T=\frac{P_{a b s}}{t_{S i N} k_{S i N}+t_{G} k_{G}} \times \frac{\ln (L / D)}{2 \pi}$. Here, $P_{a b s}$ is the absorbed optical power and $D$ is the laser spot diameter. $t_{S i N}, t_{G}$ are the thicknesses of the two materials, and $k_{S i N}, k_{G}$ are the thermal conductivities $\left(30 \mathrm{~W} / \mathrm{m} \mathrm{K}\right.$ for nitride and $5 \times 10^{3} \mathrm{~W} / \mathrm{m} \mathrm{K}$ for graphene). With a laser spot diameter of $\sim 8 \mu \mathrm{m}$, graphene absorption of $5 \%$ inside the cavity (see Figure 3(c)), and incident power of $195 \mu \mathrm{W}$, we thus expect a temperature rise of $\sim 1.3 \mathrm{~K}$. This results in a maximum frequency variation of $\Delta f=-2.7 \mathrm{kHz}$, which is an overestimate (in magnitude) since we have taken the mean membrane temperature to be that directly at the laser spot. This is, however, in excellent agreement with the measured frequency variation of $-2.2 \mathrm{kHz}$ (Figure 4(b)).

While the optical signal strength exhibits variations primarily due to its dependence on $d R / d z$, the electrical signal amplitude shows periodic variations ${ }^{32}$ mainly due to changes in the effective damping of the resonator (Figure 4(c)). In the absence of the incident optical illumination, no such 
variations are observed. Such damping variations resulting from photothermal forces have been observed in bilayer materials $^{36,37}$ and tensioned graphene drums. ${ }^{13}$ Similar effects are possible in our system, with local bimetallic expansion of the membrane breaking the system symmetry and applying a feedback force in the direction of motion. Such a force would be time delayed by the membrane thermal relaxation time and would affect both device frequency and damping. Estimates of this time constant ${ }^{32}(\omega \tau \sim 2000)$ indicate that this effect would play a significant role in damping variations but would have a negligible effect on the frequency. ${ }^{32}$ This model predicts an effective damping ${ }^{36,37}$ of $\Gamma_{e f f}=\Gamma\left(1+Q \frac{\omega \tau}{1+\omega^{2} \tau^{2}} \frac{\nabla F}{K}\right)$, where $K$ is the membrane spring constant and $\nabla F$ is the gradient in the bilayer force (w.r.t. mirror position) experienced by the membrane. The expected damping shift should thus vary as $d A(z) / d z$. However, such a model was found to have systematic deviations from our measured damping shifts. ${ }^{32}$ Thus, this is likely not the only mechanism influencing the damping of our devices, and further studies, including influence of nonlinearities, ${ }^{29,30}$ are required to understand the feedback forces in these heterostructures.

We have demonstrated the electrical actuation and detection of high $\mathrm{Q}$ silicon nitride membranes using a graphene coating to provide a conductive layer for electrical readout in a tunable Fabry-perot cavity. Optical absorption by graphene in the cavity results in position-dependent modulation of the tension in the silicon nitride, leading to discernible resonant frequency shifts due to the high $\mathrm{Q}$ of the silicon nitride/graphene resonator. Damping in silicon nitride/graphene membranes strongly depends on their position in the cavity, indicating a photothermal force on the membrane. The resonant motion of this system can thus be effectively enhanced or dampened at will and is coupled with resonant frequency control. Resonant frequencies of these high-Q systems can also separately be tuned in situ via DC gate voltage. Graphene on silicon nitride heterostructure systems thus provide for independently varying the mechanical, optical, and electrical degrees of freedom of low-mass, high-Q devices. Integrating the device with a smaller gate distance should enable utilization of the transconductance properties of graphene. Furthermore, improving the quality factor and adjusting $\tau$ via device dimensions will further enhance the photothermal interactions, potentially leading to photothermal self-oscillation of these systems which can be both detected and controlled using electrical means.

We thank the support from Cornell NanoScale Science and Technology Facility. We would like to acknowledge Dave MacNeill and Christopher B. Wallin for useful discussions. We acknowledge financial support from the Cornell Center for Materials Research and NSF Grants DMR0908634, ECCS-1001742, DMR 1120296, and AFOSR MURI, FA9550-09-1-0705.

${ }^{1}$ H. G. Craighead, Science 290, 1532 (2000).

${ }^{2}$ K. L. Ekinci and M. L. Roukes, Rev. Sci. Instrum. 76, 061101 (2005).
${ }^{3}$ J. D. Thompson, B. M. Zwickl, A. M. Jayich, F. Marquardt, S. M. Girvin, and J. G. E. Harris, Nature 452, 72 (2008).

${ }^{4}$ R. N. Dean and A. Luque, IEEE Trans. Indus. Electron. 56, 913 (2009).

${ }^{5}$ A. K. Naik, M. S. Hanay, W. K. Hiebert, X. L. Feng, and M. L. Roukes, Nat. Nanotechnol. 4, 445 (2009).

${ }^{6}$ B. Ilic, Y. Yang, K. Aubin, R. Reichenbach, S. Krylov, and H. G. Craighead, Nano Lett. 5, 925 (2005).

${ }^{7}$ J. Park, H. Qin, M. Scalf, R. T. Hilger, M. S. Westphall, L. M. Smith, and R. H. Blick, Nano Lett. 11, 3681 (2011).

${ }^{8}$ D. Garcia-Sanchez, K. Y. Fong, H. Bhaskaran, S. Lamoreaux, and H. X. Tang, Phys. Rev. Lett. 109, 027202 (2012).

${ }^{9}$ V. P. Adiga, B. Ilic, R. A. Barton, I. Wilson-Rae, H. G. Craighead, and J. M. Parpia, J. Appl. Phys. 112, 064323 (2012).

${ }^{10}$ V. P. Adiga, B. Ilic, R. A. Barton, I. Wilson-Rae, H. G. Craighead, and J. M. Parpia, Appl. Phys. Lett. 99, 253103 (2011).

${ }^{11}$ D. J. Wilson, C. A. Regal, S. B. Papp, and H. J. Kimble, Phys. Rev. Lett. 103, 207204 (2009).

${ }^{12}$ I. Wilson-Rae, R. A. Barton, S. S. Verbridge, D. R. Southworth, B. Ilic, H. G. Craighead, and J. M. Parpia, Phys. Rev. Lett. 106, 047205 (2011).

${ }^{13}$ R. A. Barton, I. R. Storch, V. P. Adiga, R. Sakakibara, B. R. Cipriany, B. Ilic, S. P. Wang, P. Ong, P. L. McEuen, J. M. Parpia, and H. G. Craighead, Nano Lett. 12, 4681 (2012).

${ }^{14}$ L. Sekaric, D. W. Carr, S. Evoy, J. M. Parpia, and H. G. Craighead, Sens. Actuator, A 101, 215 (2002).

${ }^{15}$ T. S. Biswas, A. Suhel, B. D. Hauer, A. Palomino, K. S. D. Beach, and J. P. Davis, Appl. Phys. Lett. 101, 093105 (2012).

${ }^{16}$ P.-L. Yu, T. P. Purdy, and C. A. Regal, Phys. Rev. Lett. 108, 083603 (2012).

${ }^{17}$ A. H. Castro Neto, F. Guinea, N. M. R. Peres, K. S. Novoselov, and A. K. Geim, Rev. Mod. Phys. 81, 109 (2009).

${ }^{18}$ R. R. Nair, P. Blake, A. N. Grigorenko, K. S. Novoselov, T. J. Booth, T. Stauber, N. M. R. Peres, and A. K. Geim, Science 320, 1308 (2008).

${ }^{19}$ C. Lee, X. Wei, J. W. Kysar, and J. Hone, Science 321, 385 (2008).

${ }^{20}$ J. S. Bunch, A. M. van der Zande, S. S. Verbridge, I. W. Frank, D. M. Tanenbaum, J. M. Parpia, H. G. Craighead, and P. L. McEuen, Science 315, 490 (2007).

${ }^{21}$ S. Shivaraman, R. A. Barton, X. Yu, J. Alden, L. Herman, M. V. S. Chandrashekhar, J. Park, P. L. McEuen, J. M. Parpia, H. G. Craighead, and M. G. Spencer, Nano Lett. 9, 3100 (2009).

${ }^{22}$ C. Chen, S. Rosenblatt, K. I. Bolotin, W. Kalb, P. Kim, I. Kymissis, H. L. Stormer, T. F. Heinz, and J. Hone, Nat. Nanotechnol. 4, 861 (2009).

${ }^{23}$ A. M. van der Zande, R. A. Barton, J. S. Alden, C. S. Ruiz-Vargas, W. S. Whitney, P. H. Q. Pham, J. Park, J. M. Parpia, H. G. Craighead, and P. L. McEuen, Nano Lett. 10, 4869 (2010).

${ }^{24}$ R. A. Barton, B. Ilic, A. M. van der Zande, W. S. Whitney, P. L. McEuen, J. M. Parpia, and H. G. Craighead, Nano Lett. 11, 1232 (2011).

${ }^{25}$ S. Sridaran and S. A. Bhave, Opt. Express 19, 9020 (2011).

${ }^{26}$ R. Perahia, J. D. Cohen, S. Meenehan, T. P. M. Alegre, and O. Painter, Appl. Phys. Lett. 97, 191112 (2010).

${ }^{27}$ C. Xiong, L. Fan, X. Sun, and H. X. Tang, Appl. Phys. Lett. 102, 021110 (2013).

${ }^{28}$ A. M. Eriksson, D. Midtvedt, A. Croy, and A. Isacsson, Nanotechnology 24, 395702 (2013).

${ }^{29}$ S. Zaitsev, O. Gottlieb, and E. Buks, Nonlinear Dyn. 69, 1589 (2012).

${ }^{30}$ D. Yuvaraj, M. B. Kadam, O. Shtempluck, and E. Buks, J. Microelectromech. Syst. 22, 430 (2013).

${ }^{31}$ K. H. Lee, T. G. McRae, G. I. Harris, J. Knittel, and W. P. Bowen, Phys. Rev. Lett. 104, 123604 (2010).

${ }^{32}$ See supplementary material at http://dx.doi.org/10.1063/1.4823457 for the details of the device fabrication, moving mirror setup, detection schemes, electrically and optically detected signal strength as a function of mirror position as well as calculations of cavity parameters and discussion of photothermal and absorption based models.

${ }^{33}$ D. W. Carr and H. G. Craighead, J. Vac. Sci. Technol. B 15, 2760 (1997).

${ }^{34}$ V. Singh, S. Sengupta, H. S. Solanki, R. Dhall, A. Allain, S. Dhara, P. Pant, and M. M. Deshmukh, Nanotechnology 21, 165204 (2010).

${ }^{35}$ S. Lee, V. P. Adiga, R. A. Barton, A. M. van der Zande, G.-H. Lee, B. R. Ilic, A. Gondarenko, J. M. Parpia, H. G. Craighead, and J. Hone, Nano Lett. 13, 4275 (2013).

${ }^{36}$ C. H. Metzger and K. Karrai, Nature 432, 1002 (2004).

${ }^{37}$ C. Metzger, I. Favero, A. Ortlieb, and K. Karrai, Phys. Rev. B 78, 035309 (2008). 